\title{
The Research of 3D Reconstruction Based on Single Image
}

\author{
Yong Zhang \\ School of Computer and Communication \\ Lanzhou University of Technology \\ Lanzhou 730050,China \\ yzhang@1ut.cn
}

\author{
Li Zhang \\ School of Computer and Communication \\ Lanzhou University of Technology \\ Lanzhou 730050,China \\ zlsswoaiwojia@163.com
}

\begin{abstract}
Traditional silhouette extraction algorithm is easy to cause disconnection problem, this article adopts the method of threshold segmentation and morphology repair to extract a more complete outline. The conventional SFS minimization algorithm extracted 3D information with a consistent smooth factor will make the effect is not very well, so this paper will provide a method that uses the inconsistent smooth factor to obtain a better 3D model. This paper combined the optimized silhouette information with the 3D information which obtained by improved SFS minimization method, then filter the background points, retain target points, and finally get the $3 \mathrm{D}$ model that only contains the target points. Experimental results show that the combination of this two kinds of improved algorithms can get a more real 3D model.
\end{abstract}

Keywords-SFS; 3Dreconstruction; silhouette information; smooth factor

\section{INTRODUCTION}

As an important part of digital image processing technology, the 3-D reconstruction technology has been widely applied in medical research ${ }^{[1]}$, remote sensing and telemetry $^{[2]}$ and virtual reality[3] etc. We can use a few known conditions to obtain the 3-D information of the target object by reconstructing the $3-\mathrm{D}$ model from only a single image ${ }^{[4][5]}$. In order to get a better 3-D model, the main idea in this paper is that use the 3-D reconstruction algorithm to work out 3-D information based on the more complete silhouette. Traditional edge detection technology cannot guarantee the single pixel width of the edge silhouette information ${ }^{[6]}$, which will lead to isolated or piecewise smooth edge, so the optimized method of silhouette extraction algorithm is mentioned in this paper to lay a good foundation of the 3D model reconstruction of an single image.

Currently, there are three kinds of 3-D reconstruction methods ${ }^{[7]}$ : method based on learning the statistical characteristics, method based on the shape recovery technique and the method based on the geometric information of the image ${ }^{[8]}$. The method based on learning the statistical characteristics need a large target database, it is difficult to build a perfect database ${ }^{[9]}$. The method based on the shape recovery technique obtain the 3-D model according to the priori geometry knowledge, but the geometric constraint information like parallel and perpendicular limits the scope of its application ${ }^{[10]}$. The method based on the geometric information of the image--SFX (Shape From X, X can be a solid light, shadow, silhouette ${ }^{[11]}$, texture and other information), which theory basis is the premise of the illumination and other ideal assumptions, compare with two other methods of reconstruction, the efficiency of this method is more higher, and the application scope is more widely. At present, an popular research direction is let factor $\mathrm{X}$ be shadow or shading. SFS ${ }^{[12][13]}$ (Shape From Shading) method is the research hotspot of SFX method, and the SFS method can be divided into Minimization Approach, Propagation Approach, Local Approach and Linear Approach. The Minimization Approach is a better method at present. So SFS algorithm is discussed in this paper to reconstruct $3 \mathrm{D}$ model of an image or an object.

The idea of this paper is improve the silhouette extraction algorithm first, and then on the basis of the more complete silhouette information, improve the SFS Minimization method. Finally a better 3-D model can be obtained by combining the silhouette algorithm and the SFS Minimization method. Figure. 1 shows the flow chart of this paper.

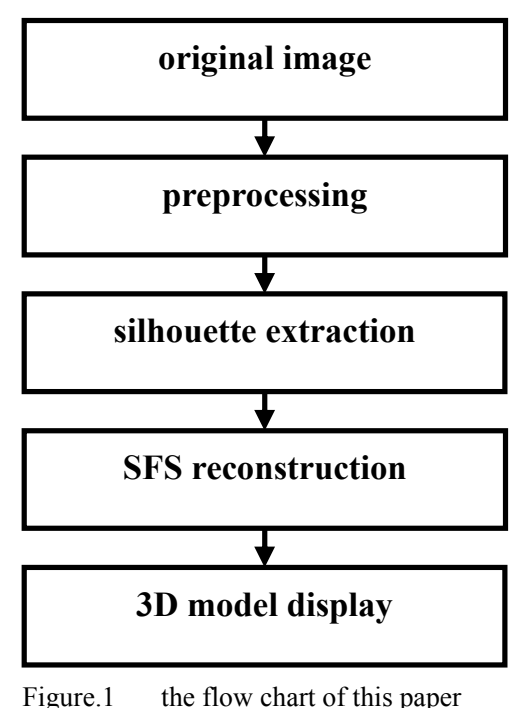

\section{Optimized Silhouette Extraction Algorithm}

\section{A. threshold segmentation}

After the preprocessing, the image can be divided into target zone and background zone. Single threshold is adopted in this paper. We assume the image is $f(x, y)$ and its grey distribution range is $\left[Z_{\min }, Z_{\max }\right]$, then we choose a threshold that in the range of $\left[Z_{\min }, Z_{\max }\right]$, finally, the output image $f_{t}(x, y)$ can satisfy the 
following equation:

$$
f_{t}(x, y)=\left\{\begin{array}{l}
1 \ldots f(x, y) \geq Z_{t} \\
0 \ldots f(x, y)<Z_{t}
\end{array}\right.
$$

The key to threshold segmentation method is how to choose a proper threshold $Z_{t}$ :

1) Divide the image into target and background with the original threshold, use the average grey of the two zone as a new threshold;

2) Use cyclic iterative method, and we can get the proper threshold $Z_{t}$ when the threshold difference of the former and later one less than the set value. In the experiment, we choose 100 as the final threshold of $Z_{t}$.

\section{B. noise repairing}

After threshold segmentation, the effect of the image always has some defect. So morphology method is adopted in this paper to repair the image, then realize noise removal.

The theory of morphology is choose proper structural element and morphological transform the image, remove noise and repairs the defect finally. A $3 \times 3$ square structural element is used in this paper. Use corrosion operation as morphological transformation to remove the small and meaningless point and object.

\section{C. silhouette information extraction}

The silhouette information extraction is realized by hollow out the inner point. Suppose the gray value of background is 0 and the gray value of object is 1 , the step of silhouette information extraction as follows:

1 ) if the pixel value of the center point is 0 , no matter the adjacent 8 pixel value is what, let the pixel value of the center point remains 0 .

2) if the pixel value of the center point is 1 , and all the adjacent 8 pixel value is 1 , turn the pixel value of the center point to 0 .

3) others, set the pixel value is 1 .

Figure. 2 shows the effect after use the optimized silhouette extraction algorithm. Figure.2a is the original grayscale image; Figure. $2 \mathrm{~b}$ is the silhouette information that get from the traditional silhouette extraction algorithm; Figure.2c is the silhouette information that get from the optimized silhouette extraction algorithm.

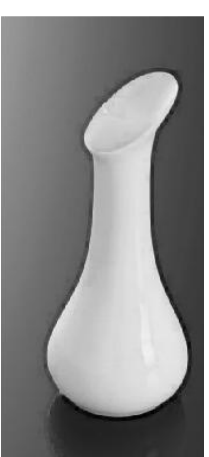

a

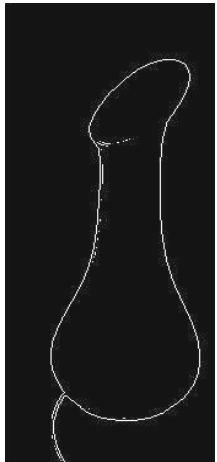

$\mathrm{b}$

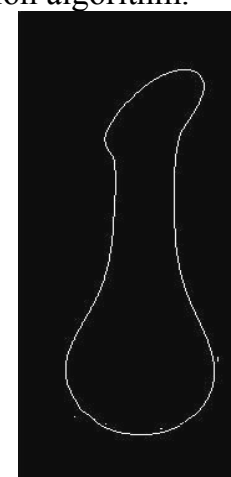

c
Figure.2 the comparison of extracted silhouette information that before and after the improvement

\section{OPTIMIZED SFS MiNIMIZATION 3D RECONSTRUCTION ALGORITHM}

We suppose the reflection model of the object is Lambert illumination model. The brightness of an image is influenced by several factors, such as light source. The material of the object, the distance between light source and object and so on. The sketch map of the Lambert illumination model is shown as in Fig.3.

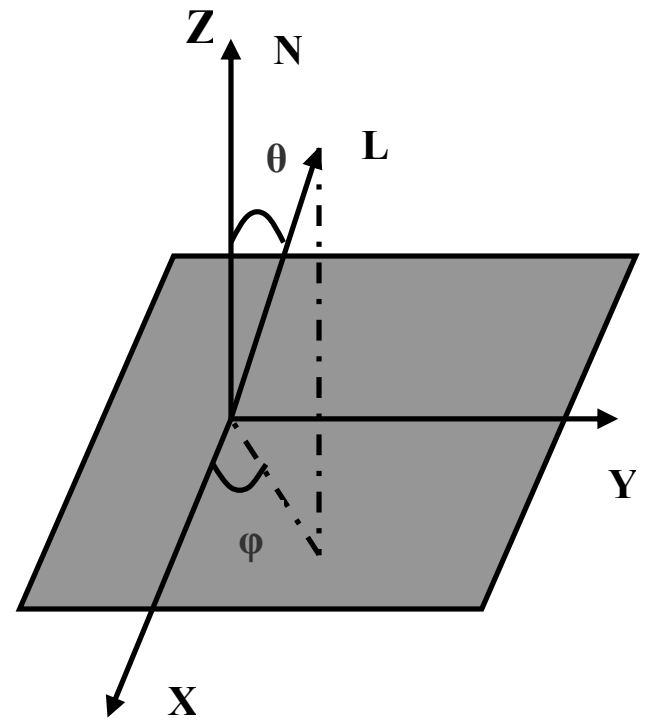

Figure.3 the sketch map of the Lambert illumination model

To simply the problem, we introduce Lambert illumination model, which satisfies the following assumptions:

1) The scene is illuminated by a single point light source which is at infinity, or the light source is uniform light;

2) The surface of the body appears equally bright from all directions and it will reflect the incident light completely.

According to the relation between reflection characteristic of the curved surface, the distribution character of light source and reflectivity of the surface, in equation (1) is the image irradiance equation:

$$
R(p, q)=I_{e} \rho \cos \angle(n, s)
$$

$R(p, q)$ Where $I_{e}$ is intensity of incident light, and $\rho$ is the reflectance coefficient, which is different materials. $\theta$ is the incident angle. $S$ represents the direction of light source and $n$ is the unit normal to the surface at point $(x, y) \cdot R(p, q)$ is the reflectance function, containing the value of the light re-emitted by the surface at point $(x, y)$ in the direction of $p$ and $q$, and $p$ and $q$ are the slope of the surface along $x$ and $y$ direction, respectively.

The normal vector of a certain point is $N(x, y)=\left(n_{x}(x, y), n_{y}(x, y), n_{z}(x, y)\right)^{T}$, for simplicity, the normal vector is denoted by 
$N=\left(n_{x}, n_{y}, n_{z}\right)^{T}$

, then the gray value of a certain pixel, the normal vector, the light direction and the reflectivity will meet the formula: $I=\rho N^{T} L$.

Suppose $\rho^{\prime}=1 / \rho$, and $\rho^{\prime} \geq 1$ then the above formula can be written as $\rho^{\prime} I=N^{T} L$.We can seen from the formula that the reflectivity $\rho^{\prime}$ and the normal vector $\mathrm{N}$ is unknown variables. In order to work out the $\mathrm{N}$, we can minimize the following energy function:

$$
E_{1}=\sum_{i \in p}\left\|\rho^{\prime} I_{i}^{T} L\right\|+\lambda \sum_{\{i, j\}}\left\|N_{i}-N_{j}\right\|^{2}
$$

$P$ is the selected area that to dealt with, $\{i, j\}$ is 4-neighborhood dot pair, $\lambda$ is called smoothness factor. The value of $\lambda$ is higher means the 3-D model has higher requirements for smoothness, vice versa.

The gradient of each point on the surface has two unknown variables, normal vector has three variables, but the pixel of each point can offer only a gray value, so SFS system is an ill system, therefore, we must apply some constraint conditions to work out the SFS problem. Generally, the constraint conditions includes: brightness constraint, smoothness constraint, integrability constraint, the gradient constraint and the unit normal vector constraint.

- brightness constraint:

$$
\begin{gathered}
\iint(I-R)^{2} d x d y \\
\iint\left(p_{x}^{2}+p_{y}^{2}+q_{x}^{2}+q_{y}^{2}\right) d x d y
\end{gathered}
$$

- integrability constraint:

$$
\iint\left(p_{y}-q_{x}\right)^{2} d x d y
$$

- gradient constraint:

$$
\text { - } \iint\left(\left(R_{x}-I_{x}\right)^{2}+\left(R_{y}-I_{y}\right)^{2}\right) d x d y
$$

- the unit normal vector constraint:

$$
\text { - } \iint\left(\|N\|^{2}-1\right) d x d y
$$

For the whole selected area that to dealt with, the energy function mentioned above applied a fixed smoothness factor $\lambda$, that is to say, for the object that will be constructed, people have no special requirements of its smoothness. Use the unique fixed smoothness factor doesn't fit the reality obviously. So this paper treat each point of the object differently, no more apply the fixed smoothness factor, but use the new energy function as follows:

$$
E_{2}=\sum_{i \in p}\left\|\rho^{\prime} I_{i}^{T} L\right\|+\sum_{\{i, j\}} \lambda_{1, j}\left\|N_{i}-N_{j}\right\|^{2}
$$

$\lambda_{i, j}$ is the smoothness factor of the $\{\mathrm{i}, \mathrm{j}\}$ dot pair. If the image is very smooth at the $\{i, j\}$ dot pair, the value of $\lambda_{i, j}$ should be slightly larger, otherwise, take a bit smaller or directly set at 0 . the value of $\lambda_{i, j}$ can first be 1 or 0 , then try to approach the median constantly, usually after a few times can achieve satisfactory results.

We can see that the function $E_{2}$ is a quadratic function, it can be work out by the iteration method. Mentioned that $\mathrm{N}$ is the unit normal vector and $\rho^{\prime} \geq 1$, so we must unitize $N$ when use iteration method each time to make sure that $\rho^{\prime} \geq 1$.

\section{EXPERIMENTAL RESULTS}

The experiment in this paper is carried on the win7 operation system, simulated by the Matlab 9.0 software. The following Figure. 4 is the effect contrast of the vase's 3 -D model between traditional method and the optimized method at the same angle of view; Figure. 5 shows the effect contrast of the vase's 3-D model between traditional method and the optimized method at the another angle of view.

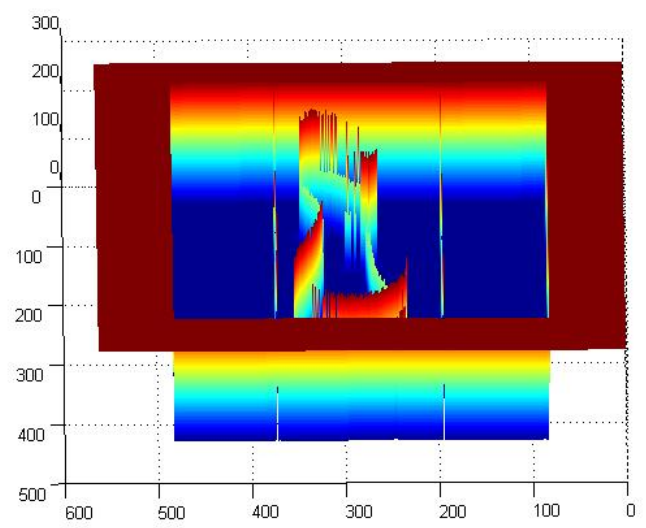

Figure.4a effect of traditional method at angle 1 of view

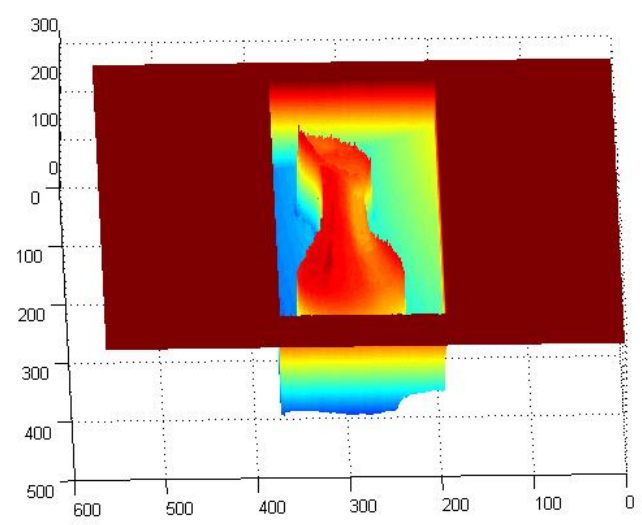

Figure. $4 \mathrm{~b}$ effect of optimized method at angle 1 of view 


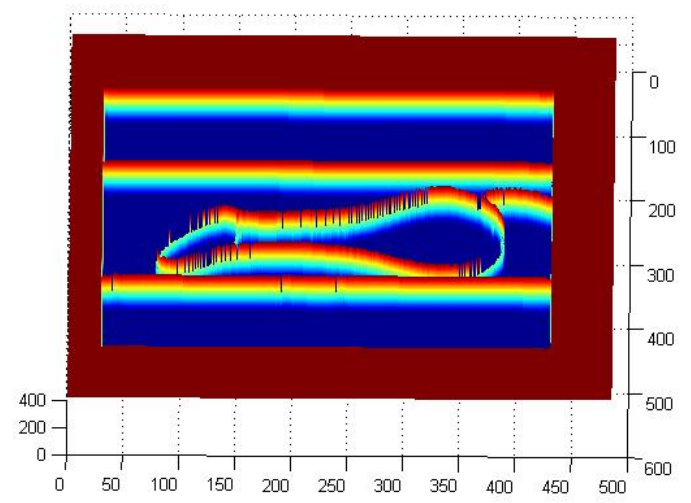

Figure.5a effect of traditional method at angle 2 of view

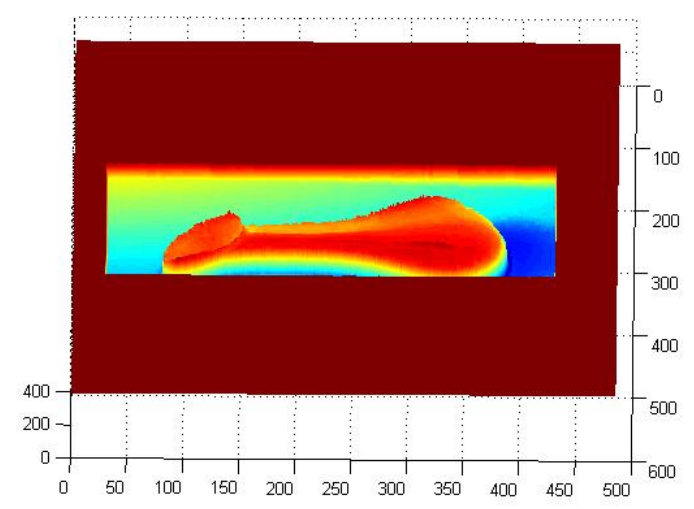

Figure.5b effect of optimized method at angle 2 of view

According to the experimental results, the error of height is compared between traditional algorithm and approved algorithm in TABLEI.

TABLE I COMPARISON OF ERROR OF HEIGHT

\begin{tabular}{cccc}
\hline \multirow{2}{*}{ algorithm } & \multicolumn{3}{c}{ error of height } \\
\cline { 2 - 4 } traditional & average & maxmum & time $/ \mathrm{s}$ \\
approved & 9.65 & 29.65 & 8.6 \\
\hline \multicolumn{4}{c}{ V. CONCLUSION }
\end{tabular}

This paper put forward an optimized silhouette extraction algorithm first, removed the defect of incomplete silhouette information. After that, using different smoothness factor improve the SFS minimization method to obtain a more real 3-D model of the object. The experimental result shows that the result of the optimized method is better than the traditional method, the detail information is more complete and the silhouette information is more clear.

\section{REFERENCES}

[1] T. Senthil Kumar, Anupa Vijai. 3D Reconstruction of Face from 2D CT Scan Images[C]. International Conference on Communication Technology and Sysem Design 2011, 30: 970-977.

[2] Xiaojing Huang, Leong Keong Kwoh. 3D Building Reconstruction and Visuali- -zation for Single High Resolution Satellite Image[C]. Geoscience and Remote Sensing Symposium, 2007. IGARSS 2007. IEEE International. 2007,7:5009-5012.

[3] Radim Dvorak, Martin Drahanaky, Filip Orsag. Object Surface Reconstruction from One Camera System[J]. International Journal of Mutimedia and Ublqultous Engineering. 2010, 5(2).

[4] Joris W. O. Salari, Gorden T. Jemwa, Hans M. Wyss. Reconstruction of the 3D Structrue of colloidosomes from a single SEM image[J]. The Royal Society of Chemistry 2011(Soft Matter). 2011,7:2033-2041.

[5] Kemelmacher-Shlizerman, Ira, Ronen Basri. 3D Face Reconstruction from a Single Image Using a Single Reference Face Shape[C]. IEEE TRANSACTION ON PATTERN ANALYSIS AND MATCHING INTELLIGENCE. 2011, 33(2):394-405.

[6] Makihara Y, Yagi Y. Silhouette extraction based on iterative spatio-temporal local color transformation and graph-cut segmentation[C]//Pattern Recognition, 2008. ICPR 2008. 19th International Conference on. IEEE, 2008: 1-4.

[7] SUN YuYang. The Research of 3D Reconstruction Technology Based on a Single Image[J]. Journal of NUCT(North China University of Technology). 2011,23(1):9-13.(in chinese)

[8] Ballan L, Cortelazzo G.M. Multimodal 3D Shape Recovery from Texture, Silhouette and Shadow Information[C]. 3D Data Processing, Visualization, and Transmission, Third International Symposium on. Chapel Hill, NC. 2006,6:924-930.

[9] Gui-Zhen HE, Xiao-jun CHENG, Cheng-quan XU. The 3D Reconstruction Based on Single Image and Accuracy Analysis[C]. 2010 International Conference on Computer Application and System Modeling(ICCASM 2010). 2010,6:208-212.

[10] Jian Wang, Defeng Chen, Tuan Zhao. Research of Three-dimensional Reconstruction Based on Computer Vision[C]. 2010 6th International conference on Wireless Communication Networking and Mobile Computing(WiCOM). 2010, 9:1-4.

[11] Hernández Esteban C, Schmitt F. Silhouette and stereo fusion for $3 \mathrm{D}$ object modeling $[\mathrm{J}]$. Computer Vision and Image Understanding, 2004, 96(3): 367-392.

[12] Quartucci Forster C H, Tozzi C L. Towards 3D reconstruction of endoscope images using shape from shading $[\mathrm{C}] / / \mathrm{Computer}$ Graphics and Image Processing, 2000. Proceedings XIII Brazilian Symposium on. IEEE, 2000: 90-96.

[13] Wu Q, Ben-Arie J. View invariant head recognition by hybrid PCA based reconstruction[J].Integrated Computer-Aided Engineering, 2008, 15(2): 97-108. 